\title{
SELECTION OF STEEL MILL AUXILIARY MOTORS AND CONTROL AS AFFECTED BY MECHANICAL FEATURES OF THE DRIVE
}

\author{
BY J. D. WRI GHT
}

\section{Abstract of Paper}

The author describes manipulators for blooming mills, which consist of side guards and lifting fingers, the former being used to guide the bloom into the proper groove in the rolls while the latter are used for turning the bloom over. The functions, mechanical layout and operation of these manipulators are described, from which conclusions are drawn as to the size and type of motors as well as the type of control best suited for driving these auxiliaries.

US SUALLY the first questions which one is likely to ask when considering the proper type of motor and control for any steel mill auxiliary machine, are, "What is its function; what is the mechanical layout, and how does the device operate?" Without a thorough knowledge of the answers to these questions one is unable to make an intelligent recommendation on the application of either motors or control.

When discussing Standardization of motors and control at a meeting of the A. I. and S. E. E. in Pittsburgh about a year ago, Mr. Friedlander, of the Carnegie Steel Co. said, "What we need is more papers descriptive of installations and operation of our mills. In such a way we would gradually get uniform ideas about general requirements at all our mills and thereby drift toward a stable system of standardization." The ProceEdings of the A. I. E. E. and A. I. and S. E. E. contain very few papers describing installations and operation of steel mill auxiliary apparatus and the writer heartily agrees with Mr. Friedlander's remarks regarding the need of such descriptive articles.

It is, therefore, the purpose of this paper briefly to describe a few steel mill auxiliary machines with particular reference to their mechanical details.

Manuscript of this paper was received February 16, 1918; released for publication February 18, 1918. 


\section{Manipulators For Blooming Mills}

Manipulators for blooming mills consist of side guards and lifting fingers. The function of the former is to guide the bloom into the proper groove in the rolls while the latter are used for turning the bloom over.

A reversing mill is usually provided with four moving side guards two on each side of the mill, one guard on the side of the mill with the operators pulpit being provided with the lifting

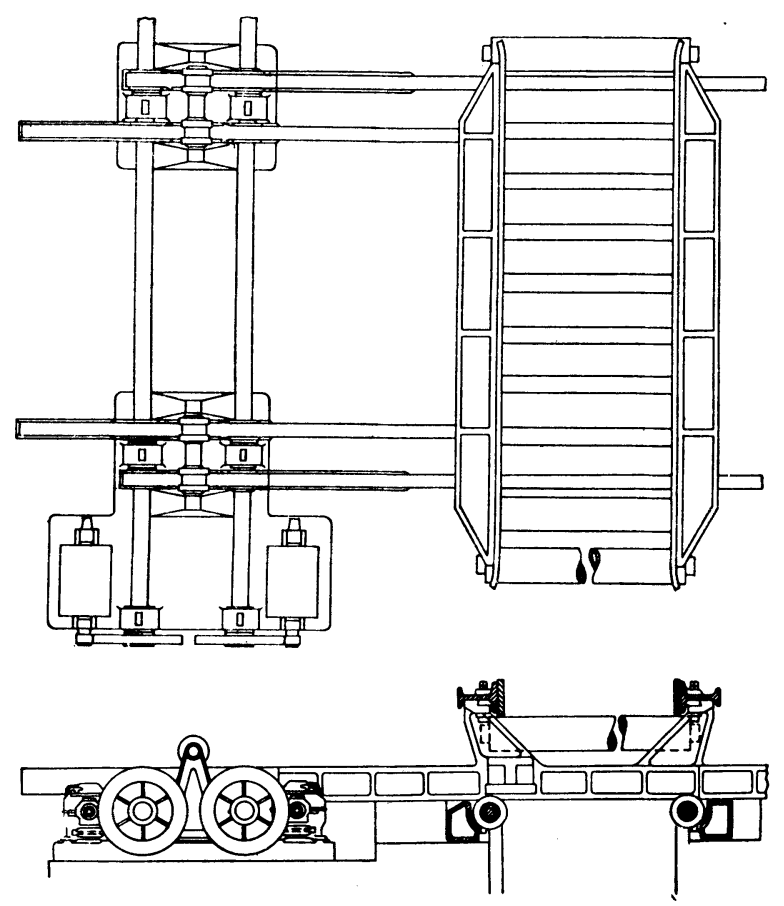

Fig. 2-Operating Mechanism for Manipulator Side Guards

fingers. Occasionally a set of lifting fingers is provided on each side of the mill. but as the bloom is usually turned only on the pulpit side, fingers are more commonly provided only on that side.

Fig. 1 is a general view of the entering side of a reversing blooming mill equipped with electrically operated manipulators. The two side guards are clearly shown and, on close inspection, the lifting fingers may be seen just inside the left hand guard.

Each side guard on the entering side of the mill is connected 
by suitable mechanical means to the corresponding guard on the delivery side, so that the right hand and left hand guards on opposite sides of the mill always have the same movement and are always in line.

Fig. 2 shows one method of mechanical connection between the motors and the side guards. Two motors, one on each side of the mill, are geared through slip clutches to a common shaft on which are mounted four pinions which engage with four racks. Two of the racks are attached to one guard on the entering side and the other two to the corresponding guard on the delivery side of the mill.

The remaining two guards on opposite sides of the mill are similarly operated by a second pair of motors. The racks are supported by rollers and mechanical stops are provided to limit their outward movement.

Fig. 3 is a view of the same mill shown in Fig. 1 but gives a more detailed view of the side guard mechanism.

As previously stated, the function of the side guards is to guide the bloom into the proper groove in the rolls. The operation of the guards during the process of rolling an ingot consists, therefore, of many fast, short movements back and forth across the table. Each guard on one side of the mill can move independently of the other and can make a stroke equal to the width of the table. It is possible therefore for the guards when moving inward to come together or against the ingot at full speed. It is the function of the slip clutch to prevent damage as a result of such action should the operator be careless enough to allow it to occur.

The motor equipment for the side guards illustrated in Figs. 1 and 3 consists of four 80-h. p. series motors, two connected in series driving each pair of guards. Satisfactory operation of the guards requires rapid acceleration and retardation, and for such service series motors are most desirable. Some installations, however, use compound wound motors, primarily because dynamic braking for stopping was desired. Furthermore, solenoid brakes are sometimes used to assist the dynamic braking. In the equipment illustrated, neither dynamic braking nor solenoid brakes are used. When a quick stop is desired the motors are plugged. The equipment is therefore extremely simple.

The control for each pair of motors is of course reversible and is arranged so that a bank of resistance is permanently connected in series with the motor armatures. This acts as a buffer and 
reduces the shock when the guards jam together or against the ingot. A one-point reversible master controller is used for each pair of motors. Limit switches are provided for cutting off power when the side guards are moved to the edge of the table. These limit switches are usually of the track type and are operated by movement of the rack. A geared switch might be used but the track type switch is much simpler and more positive in action.

The lifting fingers, which are used for turning the bloom over, are carried by a frame attached to the two inside racks connected to one of the side guards. They therefore move back and forth with the guard. See Fig. 4. The fingers are located just inside the guard and their lower ends are pinned to a yoke which
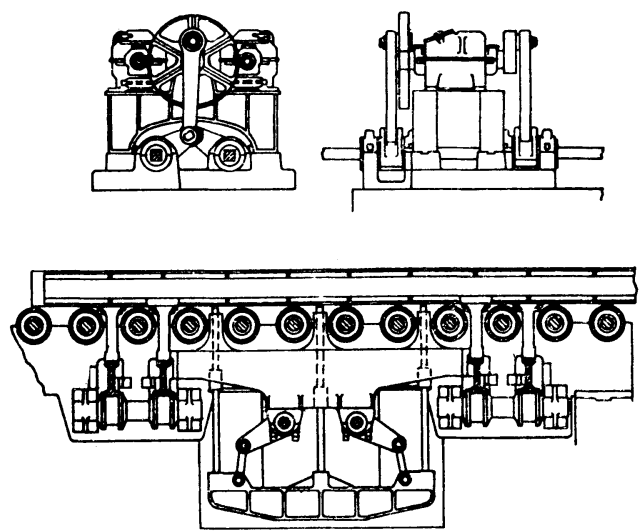

Fig. 4-Operating Mechanism for Manipulator Fingers

is suspended from two horizontal shafts by means of two links and levers. The two horizontal shafts, which are supported by bearings in the finger frame, are connected through cranks to a common driving shaft. On this shaft is mounted a large gear to which the two driving motors are geared. It is evident that one-half revolution of the main driving shaft in either direction from the position shown in Fig. 4 will produce a complete up stroke of the lifting fingers. Another one-half revolution would produce a complete down stroke and return the fingers to their orig nal position.

The motors may therefore be either reversible or non-reversible. Successful installations of each have been made. When a non-reversing equipment is used, one complete cycle 
PLATE VII.

A. I E. E.

VOL. XXXVII, NO. 3

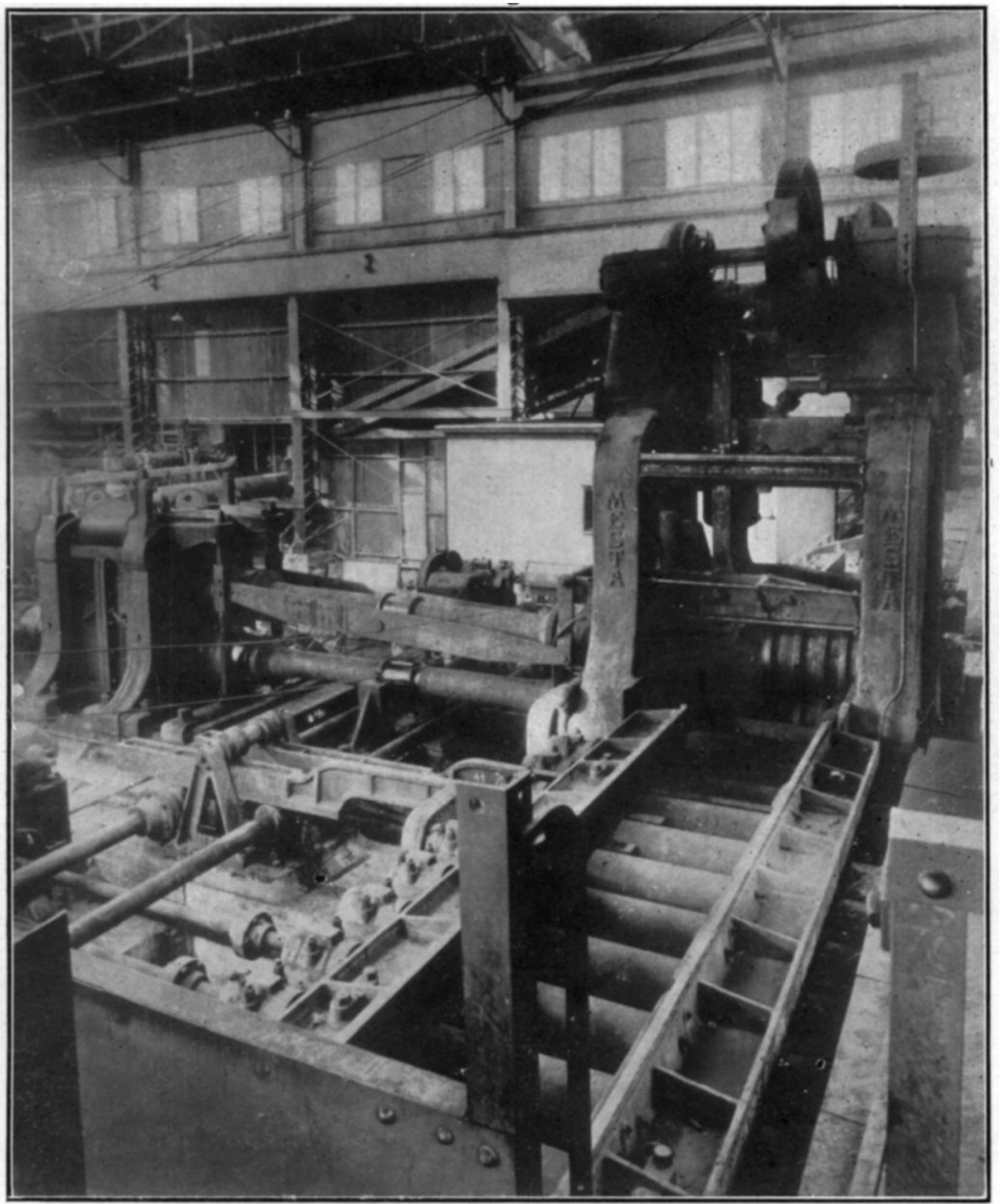

[WRIGHT]

Fig. 1-Blooming Mill with Electrically Operated Manipulators 

PLATE VIII.

A. I, E, E.

VOL. XXXVII, NO. 3

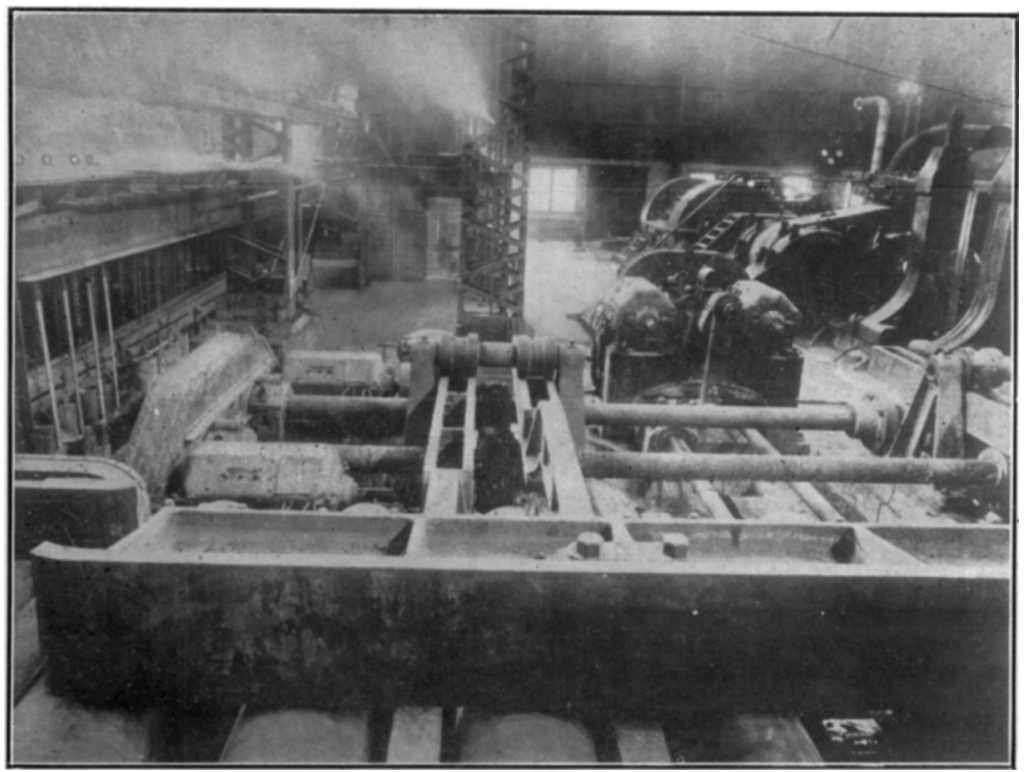

Fig. 3-General View of Operating Mechanism for Manipulator Fingers and Side Guards

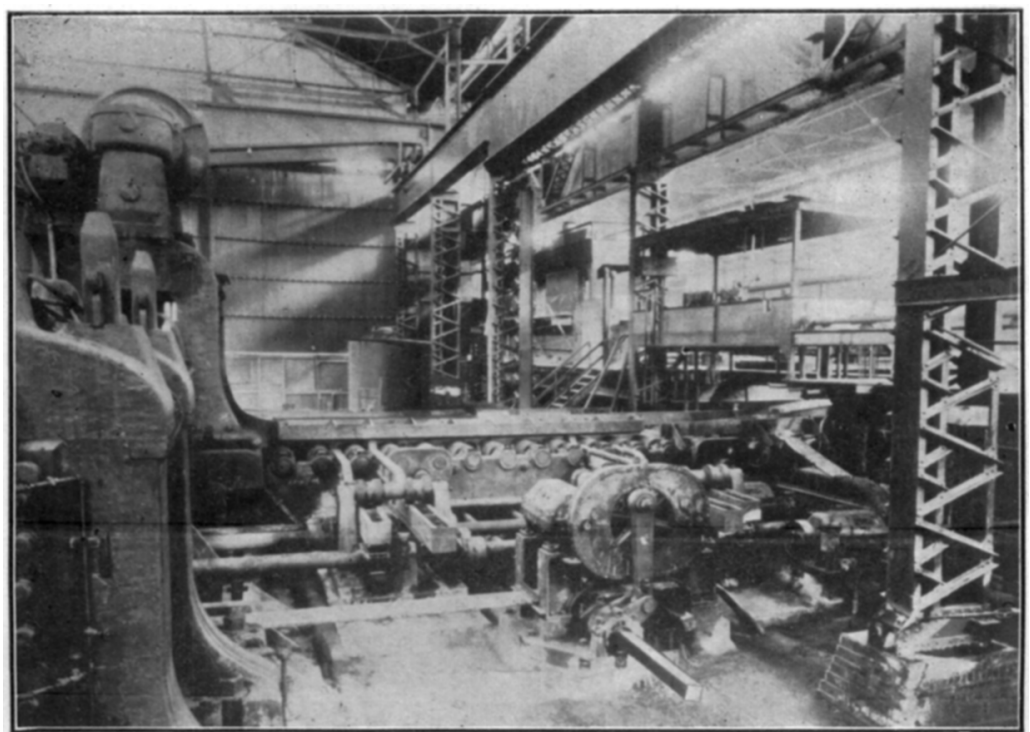

[WRIGHT]

Fig. 5-View of Blooming Mill with Electrically Operated Manipulator Fingers and Side Guards 

consists of an up and down stroke of the fingers. When a reversing equipment is used forward direction of rotation may produce the up stroke and reverse rotation the down stroke.

The motor equipment illustrated in Figs. 3 and 5 consists of two 50-h. p. series motors connected in series, operating nonreversing. For each throw of the operator's master controller a complete up and down stroke of the fingers is produced. The motors are automatically stopped at the end of the down stroke by a limit switch which cuts off power and completes a dynamic braking circuit.

The dynamic braking connections for a series motor operating non-reversing are comparatively simple. Where reversible control is used, a compound wound motor is preferable if dynamic braking is desired, as the control connections are simplified. Solenoid brakes are required if the equipment is reversible, whereas if non-reversible, no brakes are necessary. It has at times been stated that the operator should be able to control the stroke of the fingers. This of course necessitates the use of reversible control, but the movement of the fingers is so rapid that it very frequently happens that the fingers make a complete up stroke before the operator throws his master controller to the reverse position to lower them.

It has been shown that for the side guards shown diagrammatically in Fig. 2 a reversible equipment must always be provided. The motors may, however, be either series or compound wound. Dynamic braking and solenoid brakes may or may not be used. The operator's master controller may be one-point revers ble or multi-point reversible. Track type limit switches or geared limit switches to limit the movement of the side guards may be used.

For the lifting fingers in Fig. 4 it has been shown that the equipment may be either reversible or non-reversible. The motors may be either series or compound wound. Dynamic braking and solenoid brakes may or may not be used. The operators master controller may be one-point or multi-point reversible or non-reversible. The limit switch may be arranged to stop the motors when the fingers are at the end of their down stroke if the control is non-reversible, and if reversible the limit switch may be used to stop the motors with the fingers in both up and down positions.

There must, however, be one method of operating these devices which is superior to all others. What this method may be can 
be determined only from actual operating experience, and it is to the steel mill electrical engineers that we must look for the results of such experience The writer sincerely hopes that Mr. Friedlander's suggestion may be followed and that we may have more papers on installations and operation of auxiliary drives. 